\title{
Quali trasformazioni per un'inclusione reale a scuola. Disabilità e migrazione ci interrogano
}

\author{
di Valeria Friso
}

\section{Riassunto}

Il presente articolo propone riflessioni legate a traiettorie possibili che la scuola potrebbe percorrere nelle risposte a domande sempre più frequenti provenienti da alunni con disabilità figli di migranti. Rete, professionalità specializzate, valorizzazione della scuola da parte del contesto e del territorio sono alcune delle sollecitazioni che ci arrivano dalle ricerche più recenti in quest'ambito di indagine. Come renderli strumenti per una trasformazione generativa ed evitare che la scuola debba rispondere sempre con urgenza e senza percorsi condivisi?

Parole chiave: pedagogia, bisogni speciali, disabilità, migrazione, scuola.

\section{What transformations for real inclusion at school. Disability and migration question us}

\begin{abstract}
This paper proposes reflections linked to possible trajectories that schools could follow in responding to increasingly frequent questions from pupils with disabilities children of migrants. Network, specialised professionalism, school enhancement by the context and the territory are some of the suggestions coming from the most recent researches in this area of investigation. How can we make them tools for a generative transformation and avoid the school always having to respond with urgency and without shared paths?
\end{abstract}

Keywords: special needs, pedagogy, disability, migration, school.

First submission: 14/10/2021, accepted: 22/11/2021

Available online: 16/12/2021

\footnotetext{
○Università di Bologna. Corresponding author: valeria.friso@unibo.it.

Educational Reflective Practices (ISSNe 2279-9605), 2/2021 Special Issue Doi: 10.3280/erp2-Special-2021oa12912 


\section{Introduzione}

In una società come quella di oggi che sta affrontando sfide inedite, nuove ma soprattutto pervasive e che si presentano sempre più ardue da fronteggiare, la scuola è chiamata a rafforzare le azioni che rispondono alla sua responsabilità sociale di promozione della co-evoluzione di ciascun suo elemento. Oltre alle questioni già rilevanti - messe ancor più in luce dalla situazione pandemica - quali quelle legate ai nuovi analfabetismi (per esempio quelli tecnologici) e delle nuove povertà (creatasi anche a seguito della crisi economica), la pedagogia s'interroga sulla ricerca di una proposta educativa quanto più inclusiva possibile che risponda alle vecchie e alle nuove forme di emarginazione e discriminazione. I mesi che il mondo sta vivendo e attraversando stanno sicuramente richiedono dei profondi cambiamenti anche dal punto di vista sociale e culturale, perché questi forti eventi, in un certo senso, stanno destabilizzato il funzionamento ordinario sociale.

Da dove partire se non dalla scuola nella creazione di reti sociali educative volte allo sviluppo significativo della vita di ciascuna persona, affinchè quest'ultima trovi il proprio posto nella società? E non può essere proprio che sia questo il momento e il luogo idoneo per l'ideazione di nuove forme inclusive da parte del territorio e del contesto tutto?

Le scuole sono tra i soggetti che "spezzano l'isolamento delle periferie e creano rete, se la lotta si fa ricostruendo la società, le scuole possono essere, e spesso già lo sono, in prima linea, per questo vanno adeguatamente sostenute" (Tobagi, 2017, p. 337). E il sostegno alla scuola non può che nascere dal e nel territorio in cui essa è inserita: per questo motivo è una responsabilità collettiva. La partenza non può che essere, dunque, nella crescita dell'alleanza tra scuola e famiglia perché questa collaborazione evidentemente potrebbe ad ampliare i benefici per gli alunni anche in un'ottica di sviluppo di reali Progetti di Vita.

Nel presente articolo andremo a presentare come la scuola potrebbe "fare la differenza" anche, e forse ancor più, quando si impegna congiuntamente nei campi della disabilità e dell'immigrazione, due ambiti che - per studi, ricerche, politiche, scelte educative e sociali - sono sempre stati considerati in modo separato. In realtà, la compresenza di questi due mondi in un solo alunno, come vedremo, è rappresentata da percentuali non indifferenti soprattutto in determinate aree geografiche della nostra nazione.

Purtroppo, nella pratica quotidiana si constata che spesso la scuola si trova disarmata di fronte a queste situazioni e non sia sempre nelle condizioni di rispondere alle esigenze di una parte di popolazione che si presenta con un background sia migratorio e sia di disabilità. Sembra come la scuola non avesse gli strumenti, anche culturali, per attivare interventi e per creare un 
percorso quanto più inclusivo possibile che favorisca un empowerment di ciascun suo alunno per potenzialità e abilità personali, indipendentemente da cultura e deficit fisici o psichici che siano.

Di buono c'è che la recente crescita numerica di queste situazioni ha portato a interrogarsi e ad approfondire studi che hanno evidenziato quali caratteristiche non dovrebbero essere sottovalutate per un percorso inclusivo.

\section{Proposte per percorsi inclusivi per alunni con disabilità figli di mi- granti}

Iniziamo questo paragrafo affermando a gran voche che la didattica stessa si può presentare come potente strumento inclusivo (Dainese, 2019). O, se mal progettata e mal attuata, esclusivo.

In effetti, se assumiamo la seguente come buona definizione di didattica non si può non ritenerla vero mezzo possibile per cambiamenti positivi in favore dello sviluppo cognitivo e relazionale di ciascuno: "l'azione didattico/educativa può essere considerata azione mediale in quanto si colloca fra un soggetto o un gruppo di soggetti in apprendimento/formazione ed un oggetto culturale, esplicandosi nella realizzazione di mediatori, vale a dire nella costruzione delle condizioni, nella predisposizione degli strumenti e dei percorsi più adatti affinché avvengano i cambiamenti che si ritengono educativamente auspicabili" (Maccario, 2007, p. 27).

E chi sono i primi fautori per una buona azione didattica? Certamente gli insegnanti intesi come figure educative che, conoscendo la potenza della relazione, la sa interpretare, gestire, progettare, guidare e condurre. E come professionista esperto l'insegnante sa che, affinché tale relazione interpersonale e intrapersonale diventi educativa, è necessario che sia caratterizzata da almeno tre caratteristiche: intenzionalità, orientamento/direzione e progettazione (Boffo, 2020).

L'insieme fondamentale di strumenti, tecniche e metodi, spesso legati a una libera e creativa iniziativa, permetterà a ciascun allievo di apprendere attraverso il canale a lui più congeniale determinati saperi, utili per il suo futuro. E se utilizziamo la parola "futuro", come pedagogisti, non possiamo che collegarla al concetto di Progetto di vita e, quindi, anche a politiche adeguate e attente ai tempi.

Fortunatamente, da questo punto di vista, esistono documenti e linee di indirizzo che aiutano nel miglioramento anche il nostro Paese. Ci piace qui ricordare come la stessa UNESCO (2021) proponga alcuni indicatori da tenere in considerazione per permettere alle politiche educative di portare avanti compiti inclusivi: 
1. favorire l'innovazione attraverso un cambiamento inclusivo degli aspetti decisionali e politici (amministrativi, legislativi e delle risorse);

2. realizzare percorsi di formazione e aggiornamento specifico per insegnanti sull'educazione inclusiva;

3. analizzare le politiche di valutazione se sono effettivamente a favore dell'inclusione;

4. creare equilibrio organizzativo di sostegno sia dentro che fuori la scuola;

5. riconoscere e favorire l'importanza della partecipazione della famiglia nell'organizzazione delle attività educative;

6. sviluppare un curricolo attento alle diversità e alla promozione di percorsi formativi inclusivi;

7. valorizzare le risorse presenti a cui aggiungerne delle nuove per realizzare progetti inclusivi;

8. porre particolare attenzione alle fasi di passaggio e transizione dell'alunno, tra i diversi gradi scolastici e il futuro lavorativo (Unesco, 2021).

Affrontando il tema dell'inclusione legata al doppio fattore di vulnerabilità - disabilità e migrazione - è da tenere in considerazione quanto già l'uso di termini, spesso ambigui, possa creare vere e proprie barriere nella comprensione e nella costituzione di un sapere condiviso e possono diventare vere difficoltà che limitano il comune agire verso un effettivo coinvolgimento sociale di chi vive in condizioni a rischio di esclusione ed emarginazione. Il linguaggio è ciò che permette di definire la realtà che viviamo e il contesto è l'elemento principe di un qual si voglia studio sulla didattica inclusiva.

Anche rispetto a questo tema è importante che la scuola lavori in una visione di rete - interna ed esterna - divenendo agenzia che favorisce la cooperazione e la collaborazione tra i diversi enti coinvolti nella crescita di ciascun allievo. La famiglia in primis deve far parte di questa rete sociale, senza di lei si avrebbe una parziale, se non nulla, educazione e inclusione del minore. Le diversità innate di ognuno di noi in quanto esseri umani vanno a sommarsi alle differenze culturali e linguistiche di coloro che migrano, distinguendo e richiedendo risposte diverse alla società nel tentativo di soddisfare i propri bisogni. Questa rappresenta sicuramente una sfida per la scuola, intesa come ente primario di inserimento sociale.

Fortunatamente, anche se in grado diverso a seconda del territorio nazionale cui si fa riferimento, le famiglie, accanto alle scuole, possono accedere a figure professionali che facilitino non solo la relazione, ma anche la comunicazione linguistica. Queste risorse risultano essere fondamentali nella 
riuscita di una creazione di rete che deve ovviamente essere sostenuta e riconosciuta su tutto il territorio nazionale ${ }^{1}$.

Certamente resta essenziale il legame con le scelte politiche, come sopra accennato, perché l'integrazione scolastica non può esistere senza "un'integrazione abitativa, sanitaria e lavorativa. Soprattutto non esiste nessuna integrazione di allievo o famiglia straniera in questo o quell'ambito specifico, se non c'è un'integrazione diffusa, che ha per protagonisti o volontari deputati per la loro integrazione, ma anche tutte le realtà professionali e sociali, cioè tutte le persone e i gruppi che abitano un certo territorio" (Zoletto, 2007, p. 125).

È anche vero che, accanto alle scelte politiche, vi è la cultura del posto che, con le sue rappresentazioni sociali, va a definire sia la disabilità sia la migrazione. In particolare, per quanto riguarda la disabilità, è necessario ricordare che si sta parlando di un costrutto sociale, di una realtà culturalmente determinata. Infatti, il modo di rappresentarsi la disabilità varia da contesto culturale ad un altro, da un'epoca all'altra perché questa condizione di vita è interpretata, vissuta, rappresentata e quindi presa in carico in modo diverso in base al contesto di riferimento (Goussot, 2011).

Probabilmente risulta utile tornare a quanto afferma la nostra costituzione all'art. 3: "Tutti i cittadini hanno pari dignità sociale e sono eguali davanti alla legge, senza distinzione di sesso, di razza, di lingua, di religione, di opinioni politiche, di condizioni personali e sociali. Compito della Repubblica rimuovere gli ostacoli di ordine economico e sociale, che, limitando, di fatto, la libertà e l'eguaglianza dei cittadini, impediscono il pieno sviluppo della persona umana e l'effettiva partecipazione di tutti i lavoratori all'organizzazione politica, economica e sociale del Paese".

Rimuovere gli ostacoli: un compito che eleva la civiltà, ma che richiede un impegno costante, attento e anche un certo grado di creatività e innovazione.

\section{Vecchie o nuove traiettorie partecipative e trasformative a scuola?}

Per delineare delle alternative è indispensabile capire la grandezza del fenomeno che qui stiamo analizzando. Rispetto ai numeri e alle presenze di

\footnotetext{
${ }^{1}$ Questo elemento è tra i principali emersi nella ricerca coordinata dalla Prof.ssa Caldin i cui risultati sono presenti nel volume: Caldin R. (a cura di), 2012, Alunni con disabilità, figli di migranti. Approcci culturali, questioni educative, prospettive inclusivi, Napoli, Liguori; e nella ricerca coordinata dalla sottoscritta i cui risultati sono presenti in alcuni articoli tra cui: Friso, V., \& Pileri, A. (2020). Bambini migranti con disabilità nella scuola primaria. Prospettive di ricerca, «EDUCAZIONE INTERCULTURALE», 18, pp. 70-76.
} 
persone migranti nel nostro Paese sono sempre preziosi i dati offerti nei rapporti della Fondazione ISMU (Iniziative e Studi sulla Multietnicità, fonte di riferimento per le ricerche in materia di migranti a livello nazionale). È anche grazie alle ricerche di questa Fondazione che sappiamo come siano presenti nel nostro Paese circa 193 le nazioni di provenienza: la principale rimane quella rumena, seguita da quella albanese e marocchina anche se in crescita ci sono anche quella del Mali e della Guinea. I dati che ci accingiamo a presentare devono essere letti alla luce del fatto che, mentre da una parte calano gli sbarchi, dall'altra aumentano i migranti irregolari, $8,7 \%$ al $1^{\circ}$ gennaio 2018 rispetto agli 8,2\% del 2017. È una crescita costante dal 2013, anno in cui si sono conclusi gli effetti della sanatoria $\mathrm{Monti}^{2}$, e su cui bisogna porre particolare attenzione perché sono coloro che incontrano, chiaramente, più barriere nel percorso d'integrazione.

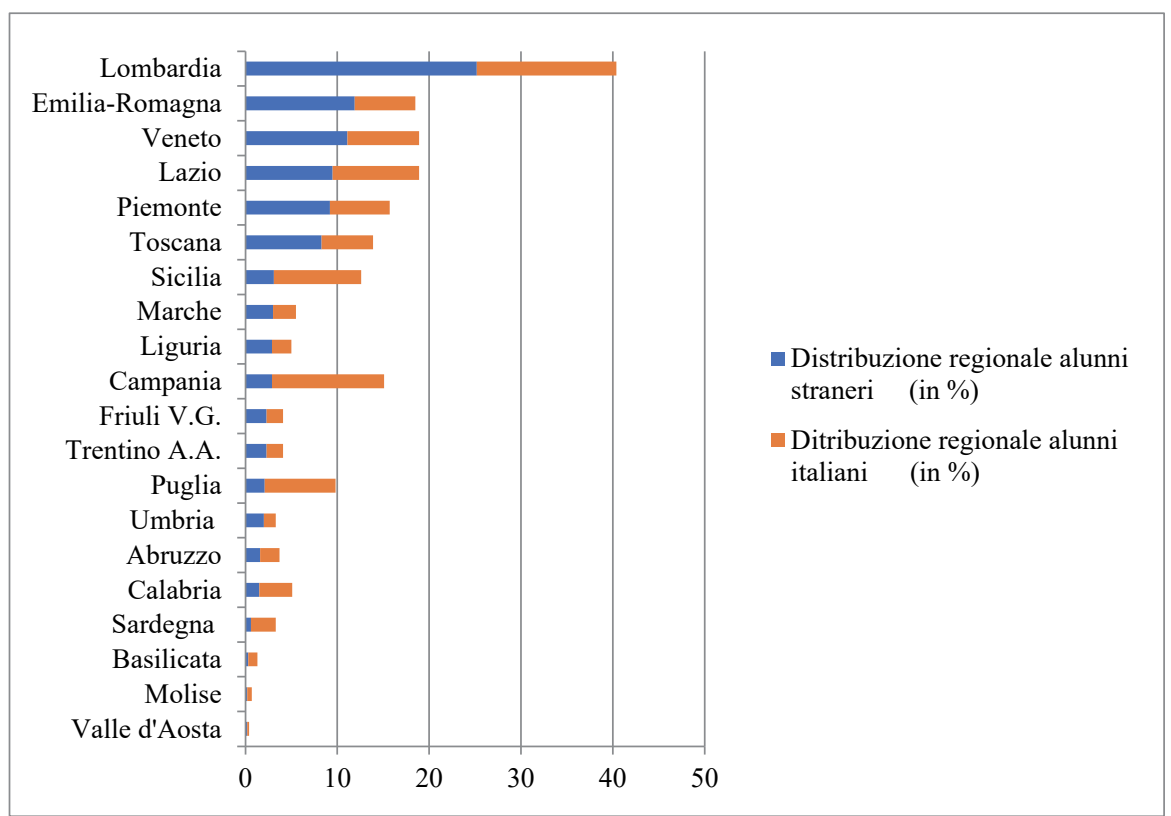

Grafico 1 - Composizione percentuale degli studenti con cittadinanza italiana e non italiana per regione - A.S. 2016/2017 - Fonte MIUR

Come è possibile vedere dal grafico 1 , gli inserimenti di alunni stranieri nelle scuole italiane maggiori sono in regioni del Nord Italia, in particolare in Lombardia (con circa 208 mila presenze) seguita da Emilia-Romagna (98

${ }^{2}$ Decreto legislativo $n^{\circ} 109 / 2012$. 
mila) e Veneto (92 mila). Guardando a questi dati possiamo affermare che la scuola di oggi è inevitabilmente frutto di un'evoluzione multiculturale.

Per quanto riguarda i numeri degli alunni con deficit invece i dati principali li si trovano nelle banche dati dell'ISTAT o direttamente nel sito MIUR. I dati più recenti in possesso rispetto alla presenza di alunni certificati ai sensi della legge-quadro 104/92 sono riferiti all'A.S. 2018/2019. Nella tabella 1 è possibile vederne il numero, rapportato al totale degli alunni, e la distribuzione sul territorio nazionale.

Tab. 1 - Totale alunni e alunni con disabilità per regione-scuole statali A.S.2018/2019 - Fonte MIUR

\begin{tabular}{lcc}
\hline Regione & Totale alunni & Totale alunni con disabilità \\
\hline Piemonte & 530.382 & 14.569 \\
Lombardia & 1.188 .581 & 40.740 \\
Veneto & 594.915 & 16.962 \\
Friuli V.G. & 144.004 & 3.646 \\
Liguria & 171.791 & 6.031 \\
Emilia-Romagna & 549.100 & 17.534 \\
Toscana & 481.118 & 14.652 \\
Umbria & 117.665 & 3.992 \\
Marche & 210.045 & 6.906 \\
Lazio & 732.994 & 24.432 \\
Abruzzo & 173.061 & 6.685 \\
Molise & 38.079 & 1.078 \\
Campania & 879.561 & 27.581 \\
Puglia & 584.982 & 17.866 \\
Basilicata & 78.054 & 1.861 \\
Calabria & 275.748 & 7.778 \\
Sicilia & 729.810 & 26.299 \\
Sardegna & 202.745 & 7.111 \\
\hline
\end{tabular}

Questi dati portano a evidenza come l'incidenza più alta degli studenti con disabilità sulla popolazione scolastica sia nelle regioni del mezzogiorno, rispetto a regioni del Centro e del Nord che sono maggiormente controbilanciate (Grafico 2).

La doppia fragilità, data dall'avere un deficit ed essere figli di migranti, ad oggi nella scuola italiana è rappresentata da una percentuale in crescita. Nell'ambito scolastico questo dato è stato - finalmente - registrato dal MIUR a partire dall'a.s. 2016/2017 e ci evidenzia come il 3,9\% degli alunni immigrati sia composto da alunni con deficit, rispetto al 2,8 \% degli studenti italiani.

Gli alunni stranieri con disabilità certificati corrispondono al $71 \%$ del totale degli alunni certificati e di questi il $43 \%$ è inserito in classi dell'Italia settentrionale. 
Grafico 2 - Totale alunni, alunni stranieri, totale alunni con disabilità e totale alunni stranieri con disabilità: composizione \% per area geografica - A.S. 2016/2017 - Fonte: MIUR

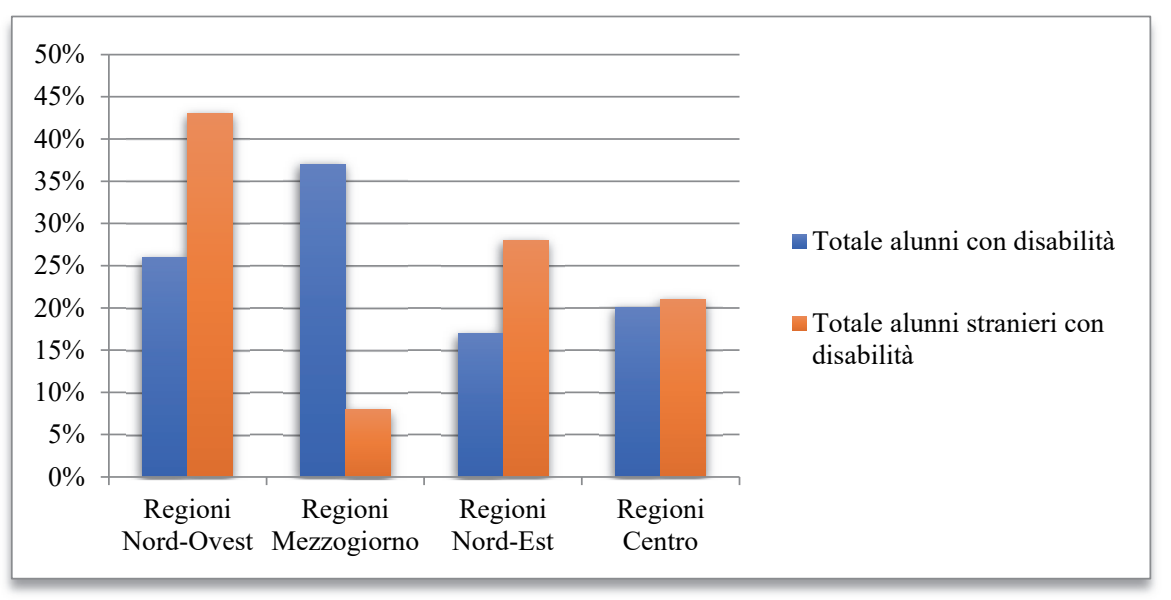

La distribuzione nazionale di questi alunni coincide con la percentuale degli stranieri presenti territorialmente a livello scolastico. Vede, infatti, ai primi posti le regioni della Lombardia, del Veneto e dell'Emilia-Romagna, seguite dal Lazio e dal Piemonte, cinque regioni che da sole accolgono il $70 \%$ degli studenti con disabilità figli di migranti.

I dati ci mostrano che, questo fenomeno, deve necessariamente essere preso sempre più in considerazione per poter offrire e garantire a questi soggetti la possibilità di ricevere un'adeguata formazione, intesa nel senso più ampio del termine, cioè nello sviluppo delle sue capacità e potenzialità.

Dato che la nostra Costituzione, volendo fare riferimento nuovamente a questo documento, afferma che "La scuola è aperta a tutti" (art. 34), non dovrebbe costituire un'eccezione incontrare scuole in cui le situazioni di fragilità sono gestite senza urgenze e con procedure condivise.

Un altro documento a cui vorremmo riferirci rispetto a questi temi è l'Index for inclusion che propone un riorientamento della sensibilità diffusa sul tema delle differenze e chiede di partire da un concetto più ampio della diversità, intesa come risorsa. Questo significa che il tema dell'inclusione va affrontato in maniera transdisciplinare, fondata su differenze e relazioni, e non più attraverso una suddivisione tradizionale di queste aree scientifiche perfettamente delimitate (Booth \& Ainscow, 2008, pp. 20-21).

Che traiettorie si possono intravedere? Le ricerche in questo campo ci propongono di lavorare, ad esempio, su alcuni strumenti di mediazione quali:

- la formazione per mediatori linguistici e ancor più per mediatori culturali: perché puntare a un'integrazione che vada verso una processualità (cioè 
che non può essere definita per sempre) e una plurilateralità (che non si limiti al solo ambito scolastico, ma coinvolga l'intera società) attraverso un dialogo attivo (nel senso di porsi in una condizione di confronto aperto e disponibile al chiarimento) di reciprocità col desiderio di capirsi e farsi capire, significa puntare verso una proposta di un percorso funzionale che non può avvenire se i principali professionisti non hanno una formazione anche specifica. L'intervento dello specialista non dovrebbe, infatti, essere rinchiuso e limitato al semplice gesto tecnico, ma sarebbe auspicabile riuscisse ad attivare i sostegni e le opportunità della rete insieme alle potenzialità della persona a cui quel gesto è riferito (nel nostro caso agli alunni con disabilità figli di migranti) per permettere a lei stessa di agire direttamente sul e nel suo contesto esistenziale.

- La valorizzazione delle figure strumentali a scuola: per permettere di fare un passo avanti che non misuri più esclusivamente la distanza dagli standard di adeguatezza, ma miri a un processo di riconoscimento della piena partecipazione alla vita scolastica da parte di ciascuno con le proprie capacità, che vengono, in questa cornice, valorizzate e rispettate fornendo a ciascuno uguali opportunità. In questo senso la scuola diventa luogo in cui chiunque si sente di essere apprezzato e che la sua partecipazione è gradita. Ad oggi esistono ancora alcuni elementi che ostacolano ancora una buona integrazione. Pensiamo al campo della disabilità nel momento in cui:

1. i bisogni dell'alunno vengono considerati solo attraverso la lettura della diagnosi clinica, proponendo quindi un approccio biomedico anziché bio-psico-sociale;

2. vi sono insufficienti risorse scolastiche;

3. il docente per le attività di sostegno viene considerato come aggiunta alla classe e non come parte della stessa;

4. la presenza non indifferente di aule di sostegno nei plessi scolastici, luoghi separati d'integrazione.

- La reale collaborazione tra scuola e territorio a partire da quella tra scuola e famiglia: in quanto un percorso educativo di un qualsiasi alunno il coinvolgimento della famiglia è essenziale per la riuscita di un progetto condiviso di crescita. Ciò da importante diventa fondamentale per quegli allievi che presentano disabilità e sono figli di migranti. Il coinvolgimento dei genitori, in questo caso specifico, dovrebbe consentire a loro e all'alunno una graduale e necessaria presa di coscienza delle proprie caratteristiche al fine di impostare un progetto di vita non alla fine delle scuole secondarie di secondo grado, ma fin dalla scuola dell'infanzia.

Sarebbe interessante, e forse solo allora, affermare di aver raggiunto un buon grado di inclusione scolastica, quando i Bisogni Educativi Speciali 
verranno considerati come una "qualsiasi difficoltà, in ambito educativo e/o apprenditivo, definita da un funzionamento problematico, anche per il soggetto, in termini di danno, ostacolo o stigma sociale, indipendentemente dall'eziologia, e che richiede educazione speciale individualizzata" (Ianes, 2005; Ianes \& Macchia 2008).

\section{Conclusioni}

L'efficacia dell'integrazione è legata al concetto di educabilità, basata su una doppia fiducia sia nel soggetto sia nell'educazione stessa (Resico, 2005a). Un'affermazione di questo tipo sembra quasi una scommessa per i contesti scolastici in cui sono presenti delle doppie fragilità come quelle su cui abbiamo proposto brevi riflessioni in questo contributo. E l'educabilità della persona, ma anche dei contesti, è il fondamento epistemologico della Pedagogia speciale la quale è chiamata a lavorare in una sempre più spiccata eterogeneità personale e culturale, che incide sui valori e i comportamenti di ciascun soggetto.

Lo sviluppo di ciascun membro della società è dato grazie a un impegno da parte di coloro che si occupano di educazione, i quali devono essere sostenuti e favoriti nel loro lavoro quasi pionieristico d'individualizzazione del percorso scolastico. Porre le fondamenta di un sistema come questo, vuol dire giungere al considerare noi stessi parte di un percorso evolutivo collettivo

Dai dati presentati si è dimostrato come una parte del contesto scolastico in crescita sia rappresentata da alunni con una doppia fragilità e un rischio più alto, nei loro confronti, di emarginazione dovuto anche all'impreparazione legislativa e formativa nonché di sensibilizzazione su un tema come quello dell'inclusione di alunni con disabilità provenienti da Paesi stranieri. E probabilmente arrivato un momento di non ritorno in cui è richiesto di accrescere gli strumenti e le competenze dei principali responsabili dello sviluppo della scuola per poter trovare risposte adeguate ai bisogni di ciascun alunno. Il dare riconoscimento a quelle risorse che, per competenze, sono diventate indispensabili per favorire un dialogo e una reciprocità, dovrebbe ormai trovare fondamento nell'impegno giuridico nazionale di voler rendere ogni persona integrata riconoscendola per le sue potenzialità e capacità. A livello internazionale e nazionale sembra chiaro che la persona in quanto tale detiene diritti inalienabili, eppure i passi da fare perché questi diritti siano riconosciuti a tutti sembrano ancora molti. Partendo da realtà locali, però, è possibile osservare e valorizzare ciò che nel piccolo molte persone favoriscono e gli esempi virtuosi sono sempre più presenti e diffusi. 
Ci piace concludere questo contributo facendo nostro il seguente augurio: "l'esperienza ci insegna che proprio nei contesti considerati maggiormente critici, dove la scommessa educativa è tutt'altro che ovvia, avvengono le scoperte più interessanti” (Frasca, 2011, p.1).

\section{Riferimenti bibliografici}

Booth, T., \& Ainscow, M. (2008). L'Index per l'inclusione, Promuovere l'apprendimento e la partecipazione nella scuola. Trento: Erickson.

Boffo, V. (2020). La relazione educativa e le competenze dell'educatore. Una riflessione per la famiglia professionale. Rivista Italiana di Educazione Familiare, 17, pp. 27-51.

Caldin, R. (a cura di) (2012). Alunni con disabilità, figli di migranti. Approcci culturali, questioni educative, prospettive inclusive. Napoli: Liguori Editore.

Dainese, R. (a cura di) (2019). La rete di relazioni a sostegno della Didattica per l'inclusione. Milano: FrancoAngeli.

Devereux, G. (2007). Saggi di etnopsichiatria generale. Roma: Armando Editore.

Frasca, V. (2011). Disabilità e migrazione: problematiche e sviluppi, Quaderni di Intercultura, III, pp. 1-9.

Friso, V., \& Pileri, A. (2020). Bambini migranti con disabilità nella scuola primaria. Prospettive di ricerca. Educazione Interculturale, 18, pp. 70-76.

Goussot, A. (a cura di) (2011). Bambini "stranieri" con bisogni speciali. Saggio di antropologia pedagogica. Roma: Aracne.

Ianes, D. (2005). Bisogni educativi speciali e inclusione. Valutare le reali necessità e attivare tutte le risorse. Trento: Erickson.

Ianes, D., \& Macchia, V. (2008). La didattica per i BES. Trento: Erickson.

Maccario, D. (2007). Le nuove professioni educative. La didattica nei servizi socioeducativi e assistenziali. Roma: Carrocci.

Resico, D. (2005a). Ai confini dell'educabilità. Pedagogia speciale e relazione d'aiuto, Milano: FrancoAngeli.

Tobagi, B. (2017). La scuola salvata dai bambini. Milano: Bur Rizzoli.

Unesco (2021). "EDUCAZIONE ALLO SVILUPPO SOSTENIBILE UNA TABELLA DI MARCIA" \#ESDFOR2030. http://www.unesco.it/it/TemiInEvidenza/Detail/52 (consultato il 6/10/2021).

Zoletto, D. (2007). Straniero in classe. Pedagogia dell'ospitalità. Milano: Raffaello Cortina Editore. 